\title{
Injury Type, Injury Severity, and Repeat Occurrence of Alcohol-Related Trauma in Adolescents
}

\author{
Ronald F. Maio, Joel Portnoy, Frederic C. Blow, and Elizabeth M. Hill
}

\begin{abstract}
Injury associated with alcohol use is a significant problem among adolescents; however, routine evaluation of alcohol use in this population is not conducted. The purpose of this study was to compare Injured adolescents presenting to an emergency room with a positive serum alcohol concentration (SAC+) with those injured adolescents with a negative serum alcohol concentration (SAC-). Data were collected retrospectively on 176 injured patients, between the ages of 13 and 18 , consecutively admitted to a university hospital from January 1, 1989-December 31, 1990. Information collected included mechanism and severity of injury, outcome, SAC, length of stay, paychiatric history, prior or subsequent admission for injury, and hospitel charges. Of those tested with an SAC, more than one-third had a positive SAC. Patients with positive SACs had a greater probability of having a psychiatric history and more frequently had a prior or subsequent injury. Furthermore, only $34 \%$ of SAC+ patients were referred for counseling. The results indicate that a SAC should be obtained on all adolescents admitted for trauma, that adolescents presenting with injuries and a positive SAC should be referred for alcohol and psychiatric assessment, and that injured adolescents may be at increased risk for repeat injuries in the future.

Key Words: Ethanol, Injury, Adolescents, Injury Prevention.
\end{abstract}

\begin{abstract}
ALCHOL USE HAS been noted to be a contributing
factor in many injuries sustained by adolescents and young adults. ${ }^{1-4}$ In fact, the major preventable health problems of adolescents fall into two main categories: (1) injuries that kill and disable and (2) emerging lifestyles that affect adolescent health for many years. ${ }^{5}$ Identifying injured patients in the Emergency Department (ED) who have positive blood tests for alcohol has important clinical implications regarding their immediate evaluation and treatment. ${ }^{6-11}$ In addition, the presence of a positive alcohol test may indicate that the injured patient has a substance abuse problem that could place him or her at increased risk both for future injury and for other deleterious effects on long-term health and social status. ${ }^{12}$
\end{abstract}

From the Section of Emergency Medicine, Department of Surgery, University of Michigan (R.F.M., J.P.); and the Department of Psychiatry, University of Michigan Alcohol Research Center (F.C.B., E.M.H.), Ann Arbor, Michigan.

Received for publication March 24, 1993; accepted August 23, 1993

This study was supported in part by the National Institutes of Health Training Grant HL0690-12 and by the National Institute on Alcohol Abuse and Alcoholism Center Grant P50-AA07378. Preliminary results were presented at the Research Society on Alcoholism Meeting, San Diego, CA, June 13-18, 1992

Reprint requests: Ronald F. Maio, D.O., University of Michigan Medical Center, Taubman Center, TC B1354-0303, 1500 East Medical Center Drive, Ann Arbor, MI 48109-0303.

Copyright $(1994$ by The Research Society on Alcoholism.
The American College of Emergency Physicians has recognized and emphasized the importance of identifying and treating the behavioral and emotional components of acute injury. ${ }^{13}$ The majority of previous research on alcohol and injury has examined a broad age range of patients. Only one previous study has evaluated adolescents admitted to hospital following trauma. ${ }^{1}$ This study did not evaluate prior or subsequent injury, whether the patient was referred for counseling during or after the admission, or the presence of any psychiatric problems. The purpose of the present study is 2-fold: (1) to compare data on injured adolescents admitted to hospital following alcohol-related injuries to data from those admitted after nonalcohol-related injuries; and (2) to determine the relationship of repeat trauma admissions with positive serum alcohol concentrations (SACs).

\section{METHODS}

This is a retrospective cohort study. The study sample was selected from all patients admitted to University of Michigan Medical Center's (UMMC) for trauma who were 13-18 years old. Trauma patients were defined as those with one or more ICD-9 diagnosis from 800-959.9 (range of ICD-9 trauma codes). The study period was from January 1 , 1989 through December 31, 1990. Appropriate cases were identified via the UMMC Medical Information Services. The medical records for these cases $(n=300)$ were obtained, and information was abstracted. Eightyseven cases arrived at UMMC $>8 \mathrm{hr}$ after injury and were excluded. Forty cases were readmissions after recent surgery and were excluded. The medical records for 7 cases could not be found. Of the remaining 176 cases, 106 had SACs obtained. These 176 cases formed the final study sample. If a case was transported from another hospital and had a documented SAC result from the sending hospital, that SAC was used. Otherwise the SAC used was the earliest documented SAC drawn at the UMMC.

During the study period, there was no specific ED policy or triage criteria for adolescents or adults regarding alcohol and drug testing in general or specifically for injured patients. Attending physicians, at that time, included those with residency training in Internal Medicine, Surgery and occasionally, Emergency Medicine or Pediatrics. Adolescent trauma patients in the ED were evaluated and treated by attending staff or surgical house officers assigned to the ED or on consulted services. Patients were categorized into two groups: SAC-negative (SAC-), e.g., patients with a SAC of 0 ; and SAC-positive $(\mathrm{SAC}+)$, e.g., patients with $\mathrm{SAC}>0$. Patient gender, age, and the mechanism of injury [motor vehicle crash (MVC)/other] was also collected. The presence of a positive psychiatric history (documented treatment for substance abuse, depression, or suicide attempt) and prior or subsequent injury (another admission for trauma to UMMC within 5 years prior or 1 year subsequent to the admission studied) was also noted. Outcome measures were length of stay (LOS), cost (amount billed to the patient including all emergency 
room charges, hospital room and board, diagnostic procedures, operative procedures and equipment), and survival (discharged alive from hospital or died). Physician fees were not included.

Injury severity was calculated based on the Abbreviated Injury Scale (AIS)-1985 version. ${ }^{14}$ This scale classifies injuries in each of eight body regions using an ordinal scale of $1-6$, with 1 as least severe and 6 as most severe. The actual measure of injury severity used in the study was the Injury Severity Score (ISS). ${ }^{15}$ This score is calculated by summing the squares of the AIS scores of the three ISS body regions having the highest AIS scores. The AIS and ISS scores were calculated using TRI-CODE ${ }^{\infty}$ (Tri-Analytics, Bel-Air, MD), a computerized injury severity scoring program. The AIS and ISS are validated, widely used measures of anatomic injury severity. Previous or current psychiatric problems were determined by noting if the medical records reflected ongoing or previous history of psychiatric treatment (including substance abuse). Previous admissions for trauma were determined by a combination of two methods: (1) computer search of UMMCs medical records for past trauma admissions of study patients for years 1985-1988 inclusive; and (2) noting the mention of a previous injury on the medical record. Subsequent trauma admissions were determined by computer search of medical records for the time period from January 1, 1989-December 31, 1991.

Categorical analysis of dichotomous measures (gender, motor vehiclerelated trauma vs. other, mortality, ISS $>=25$, psychiatric history, and previous or subsequent injury) was conducted using $\chi^{2}$ tests; continuous, normally distributed variables (age, cost, and LOS) were analyzed using two-tailed $t$ tests; and ordinal variables (ISS) were analyzed using the Mann-Whitney U nonparametric test. Statistical significance was set at $p<0.05$.

This study was approved by the University of Michigan Committee to Review Grants for Clinical Research and Investigation Involving Human Beings.

\section{RESULTS}

Table 1 shows a comparison between patients who were tested for SACs and those who were not for gender, age, ISS, ISS $>=25$, MVC as mechanism, mortality, cost, LOS, and prior or subsequent trauma admission. Patients not having a SAC performed were younger, had lower injury severity and mortality, and were less likely to be in a MVC. Adjusting for multiple comparisons using Bonnferroni's correction resulted in the loss of statistical significance for age, LOS, and cost.

One hundred and six patients had SACs done. Thirtyeight had positive SACs. These SACs ranged from 0.0 to $287.7 \mathrm{mg} / \mathrm{dl}$. Table 2 shows a comparison between SAC+

Table 1. Descriptive Characteristics of SAC Tested Vs. SAC Not Tested Patients

\begin{tabular}{lccc}
\multicolumn{1}{c}{\begin{tabular}{c} 
SAC No \\
\multicolumn{1}{c}{ Variable }
\end{tabular}} & $\begin{array}{c}\text { SAC Yes } \\
(n=106)\end{array}$ & $\begin{array}{c}\text { SAC } \\
(n=70)\end{array}$ & $\rho$ Value \\
\hline Sex & & & NS \\
$\quad$ Male & $75 \%$ & $77 \%$ & \\
Female & $25 \%$ & $23 \%$ & \\
Age (mean) & 16.5 & 15.8 & $<0.05$ \\
ISS (median) & 13.0 & 5.0 & $<0.05$ \\
ISS > = 25 & $17.9 \%$ & $4.3 \%$ & $<0.05$ \\
Motor vehicle & $69 \%$ & $21 \%$ & $<0.05$ \\
Mortality & $10.5 \%$ & $0.0 \%$ & $<0.05$ \\
Cost (mean) & $\$ 22,182$ & $\$ 14,429$ & $<0.05$ \\
LOS (mean) & 11.5 & 7.4 & $<0.05$ \\
Prior/subsequent trauma & $24 \%$ & $19 \%$ & NS \\
$\quad$ admissions & & & \\
Positive psychiatric history & $10 \%$ & $4 \%$ & NS \\
\hline
\end{tabular}

NS, not significant $(\rho>0.05)$.
Table 2. Descriptive Characteristics of SAC+ Vs. SAC- Patients $(n=106)$

\begin{tabular}{lccc}
\hline \multicolumn{1}{c}{ Variable } & $\begin{array}{c}\text { SAC+ } \\
(n=38)\end{array}$ & $\begin{array}{c}\text { SAC- } \\
(n=68)\end{array}$ & $p$ Value \\
\hline Sex & & & NS \\
$\quad$ Male & $82 \%$ & $71 \%$ & \\
$\quad$ Female & $18 \%$ & $29 \%$ & \\
Age (mean) & 16.7 & 16.3 & NS \\
ISS (median) & 12.5 & 13.0 & NS \\
ISS > = 25 & $8 \%$ & $24 \%$ & $<0.05$ \\
Motor vehicle & $68 \%$ & $69 \%$ & NS \\
Mortality & $3 \%$ & $15 \%$ & $<0.05$ \\
Cost (mean) & $\$ 20,191$ & $\$ 23,295$ & NS \\
LOS (mean) & 11.9 & 11.3 & NS \\
Prior/subsequent trauma & $21 \%$ & $11 \%$ & NS \\
$\quad$ admissions & & & \\
Positive psychiatric history & $24 \%$ & $2 \%$ & $<0.05$ \\
\hline
\end{tabular}

NS, not significant $(p>0.05)$.

Table 3. Distribution of Patients with Prior or Subsequent Trauma Admissions

\begin{tabular}{llll}
\hline & SAC+ & SAC- & \\
\hline $\begin{array}{l}\text { Prior/subsequent trauma } \\
\text { admissions (t) }\end{array}$ & $8(21 \%)$ & $8(11 \%)$ & 16 \\
$\begin{array}{l}\text { Prior/subsequent trauma } \\
\text { admissions (-) }\end{array}$ & $30(79 \%)$ & $60(89 \%)$ & 90 \\
& 38 & 68 & 106 \\
\hline
\end{tabular}

$\chi^{2}=1.641, p=0.200$

and SAC - groups for gender, age, ISS, ISS $>=25$, MVC as mechanism, mortality, cost, and LOS. Patients with a negative SAC had significantly higher ISS scores and mortality.

About one-fourth of the SAC+ patients had a previous psychiatric history compared with almost none of the SAC- group. Chart notation indicated that the majority of these patients had substance abuse problems ( 8 of 11 ). Only $34 \%$ of patients with a positive SAC received counseling or were referred for counseling during admission. Although average age did not differ significantly between groups, $17 \%$ of the SAC+ patients were 15 years of age or under compared with $30 \%$ for the SAC- group $\left(\chi^{2} 2.925\right.$, $p=0.087)$. Even though SAC+ patients were generally older teens, one patient with a positive $S A C$ was 13 years old.

Table 3 shows the distribution of SAC+ and SACpatients with prior or subsequent trauma admissions. Although almost twice the percentage of SAC+ patients compared with SAC- patients $(21 \%$ vs. $11 \%)$ had a prior or subsequent trauma admission, this difference was not statistically significant.

\section{DISCUSSION}

Although injury is the major cause of death for adolescents, and alcohol has been associated with those injuries, only one study has specifically addressed the alcohol/ injury relationship in adolescents. ${ }^{1}$ Emergency physicians need to identify adolescent trauma patients with positive blood alcohol tests to ensure optimal evaluation and emergency treatment. No less important is the emergency physician's opportunity for injury prevention and intervention in these adolescent trauma patients by identifying 
behavioral problems that may lead to further injury or other noninjury disease. ${ }^{16}$

This study noted that patients as young as 13 years of age may have a positive SAC. Of those tested, a positive or negative SAC could not be differentiated based independently on either gender, age, or mechanism of injury. More severely injured admitted patients were not more likely to have a positive SAC, although they were more likely to be tested. Thirty percent of all the adolescents tested had positive SACs. This study found that $18 \%$ of female admitted trauma patients had a positive SAC. Almost $20 \%$ of the patients with a positive SAC were 15 years of age or younger.

Also, adolescents admitted after injury with a positive SAC were more likely to have a psychiatric history and may be more likely to have a prior or subsequent admission for trauma. Mortality for the SAC+ group was 3\% compared with a $15 \%$ mortality for the SAC-group. Even though the median ISS scores were essentially the same, the percentage of ISS $>25$, indicating severe trauma, was almost three times higher in the SAC - group and is most likely the reason for this group's higher mortality. Because no data were collected regarding the severity of forces causing the injury, it was impossible to evaluate any potentiating effect on injury from alcohol. This is a problem that illustrates the limitations of using hospital-based injury data as opposed to population-based data. ${ }^{17,18}$

Admitted injured adolescents with a positive SAC are more likely to have a psychiatric history, and this psychiatric history is most likely to be related to substance abuse. It is particularly distressing that only $35 \%$ of the surviving patients admitted with a positive SAC received or were referred to counseling. One other study, including all ages, noted no referrals for substance abuse among a group of trauma patients admitted with positive blood alcohol tests. ${ }^{19}$ Whether this lack of appropriate psychiatric referrals reflects the insensitivity of clinicians, institutional procedural issues, patients' attitudes, or concerns regarding reimbursement will require further study.

Resuits appeared to indicate that more $\mathrm{SAC}+$ patients had prior or subsequent trauma admissions than SACpatients in this sample. However, the differences found were not statistically significant and therefore could be due to chance alone. Because of the small numbers of prior/subsequent trauma admissions, we did not analyze prior trauma admissions and subsequent trauma admissions separately. Future studies, requiring in depth interviews, should be done to better assess prior and subsequent trauma admissions.

In our study, SAC - patients had a higher mortality and would be more likely to have a lower subsequent admission rate, because the denominator was not adjusted for these deaths. It also was not possible to determine accurately if those prior and subsequent admissions were associated with alcohol use.

As with the SAC and psychiatric history relationship previously discussed, one cannot infer if alcohol use, in general, predisposes individuals to injury or that those patients with a greater predisposition to injury are more likely to use alcohol. The combined prior/subsequent admission rate for both SAC groups of $15 \%$ and may imply that admission to a hospital following an injury is a marker for individuals who are risk takers and may be more prone to injury. Other studies suggest that an emergency visit for injury is more strongly related to alcohol abuse and previous injury than with alcohol test results. ${ }^{20-22}$ Other investigators have stated that an SAC (200 $\mathrm{mg} / \mathrm{dl}$ ) or greater in an ED patient, whether trauma- or nontrauma-related, is indicative of long-term problems with alcohol. ${ }^{23,24}$ Regardless of the causal relationship between alcohol and injury, adolescent patients admitted for injury who have a positive SAC should be counseled on their potential increased risk of future injury and future substance abuse.

Despite low testing rates among admitted trauma patients of all ages, which is concordant with another study, ${ }^{19}$ it is disconcerting that in this study only slightly more than one-third of all admitted adolescent trauma patients had SAC testing conducted. Apparently, clinicians believe they can accurately screen patients in need of SAC testing. However, examination of variables that can easily be assessed on admission to the ED (age, gender, mechanism of injury) showed no significant differences between the $\mathrm{SAC}+$ group and the SAC- group. This finding suggests that, among adolescents, as with older injured patients, initial clinical assessment alone may not identify all trauma patients with a positive SAC.

These data also demonstrate that even the youngest adolescents may have alcohol-associated trauma admissions. Particularly noteworthy is the large percentage of patients (20\%) who were age 15 and under in the SACpositive group. The only other study of adolescent trauma patients did not include patients under the age of 16 because of previously reported extremely low rates of positive alcohol tests among young adolescents. ${ }^{1}$ However, one study investigating substance abuse in 9- to 17-yearolds noted that $56 \%$ of those studied reported occasional to regular use of alcohol. ${ }^{28}$ Thus, ED personnel cannot universally assume that younger adolescents will have a negative SAC and therefore do not require testing.

Several limitations of this study require mention. The small sample size limits the ability to detect statistically significant differences. To find a statistically significant difference of $10 \%$ for prior or subsequent trauma admissions ( $21 \%$ for SAC+ and $11 \%$ for SAC-) using two-sided testing at an $\alpha$ of 0.05 would require a total of 440 patients; our study with 106 patients has a power of 0.30 to detect a difference of $10 \%$.

Selection bias is another potential drawback of this study. Only admitted patients were evaluated. The vast majority of adolescent trauma patients presenting to the UMMC ED are not admitted, yet their injuries may cause 
significant morbidity and financial cost. Selection bias regarding SAC testing is also a problem, but two of our main variables of interest, subsequent and prior admissions and psychiatric history, were randomly distributed between tested and nontested individuals.

The relationships among alcohol abuse, psychiatric history, and prior and future injury may be much different in nonadmitted injured adolescents compared with their admitted counterparts. Perhaps the incidence of a positive SAC is greater in nonadmitted than admitted injured adolescents. Indeed, the population of nonadmitted adolescents may represent an at-risk population whose ED visit provides an excellent opportunity for intervention. Furthermore, we studied patients who were admitted to a university medical center. The findings, therefore, may not be representative of adolescent trauma patients presenting to community hospitals. Prior and subsequent trauma admissions were determined only from hospital records. It is quite probable that patients in our study population may have been treated for injuries at other hospitals. Therefore, this study's estimate of prior and subsequent trauma admissions, for adolescent patients undergoing SAC testing, may be conservative.

In spite of these preceding limitations, this study expands the limited knowledge base in the area of adolescents, injury, and alcohol. Evaluation of the injured adolescent for alcohol use and abuse is critical to ensure appropriate short- and long-term hospital-based care. ED personnel have an excellent opportunity to identify individuals at high risk for future injury and to implement strategies that will help decrease this risk. This study showed that it may be impossible to screen appropriately for patients with a positive SAC by patient characteristics alone and that adolescents in the youngest age range may have a positive SAC. This study also demonstrated that adolescents admitted for trauma with a positive SAC are more likely to have a psychiatric history and may be more likely to have prior or subsequent admissions for trauma. Finally, counseling or referral for adolescent patients with a positive SAC is infrequently done.

Injury and associated alcohol abuse are taking a devastating toll on today's youth. This study suggests that health care professionals caring for acutely injured adolescents need to do a better job of evaluating and treating all components of the patient's problem. Further studies are needed to determine the most accurate and efficient methods for evaluating adolescent trauma patients for alcohol use, and for providing appropriate counseling and intervention.

\section{REFERENCES}

1. Hicks BA, Morris JA, Bass SM, et al: Alcohol and the adolescent trauma population. J Pediatr Surg 25:944-949, 1990
2. Friedman IM: Alcohol and unnatural deaths in San Francisco youths. Pediatrics 76:191-193, 1985

3. Paulson JA: The epidemiology of injuries in adolescents. Pediatr Ann 17:84-96, 1988

4. Williams AF, Pent MA, Crouch DJ, et al: Drugs in fatally injured male drivers. Public Health Rep 100:19-25, 1985

5. U.S. Department of Health and Human Services, Public Health Service: Healthy People 2000: National Health Promotion and Disease Prevention Objectives. Washington, D.C., Department of Human Health and Services, 1990, pp 16-18

6. Lowenfels AB: Alcohol and trauma. Ann Emerg Med 13:10561060,1984

7. American College of Surgeons: Advanced Trauma Life Support. Chicago, American College of Surgeons, 1988, pp 113-125

8. Ross S: Early Care of the Injured Patient, chap 14, The Abdomen, ed 4. Philadelphia, B. C. Decker, Inc., 1990, pp 159-175

9. Ducker TB, Aryanpur J: Early Care of the Injured Patient, chap 10, Central Nervous System, ed 4. Philadelphia, B. C. Decker, Inc., 1990, pp 107-125

10. Krome RL: Emergency Medicine: Comprehensive Study Guide, ed 2. New York, McGraw-Hill, 1985, pp 825-830

11. Marx JA: Emergency Medicine: Concepts and Clinical Practice. St. Louis, C. V. Mosby Company, 1992, pp 380-408

12. Sims DW, Bivins BA, Obeid FN, Horst HM, Sorensen VJ, Fath JJ: Urban trauma: A chronic recurrent disease. J Trauma 29:940-947, 1989

13. American College of Emergency Physicians: The emergency physician's role in behavioral emergencies. Ann Emerg Med 972:163164,1987

14. American Association for Automotive Medicine: The Abbreviated Injury Scale (AIS)-1985 Revision. Des Plaines, IL, American Association of Automotive Medicine, 1985

15. Baker SP, O'Neill B, Haddon W Jr, et al: The Injury Severity Score: A method for describing patients with multiple injuries and evaluating emergency care. J Trauma 14:187-196, 1974

16. U.S. Department of Health and Human Services, Public Health Service: Healthy People 2000: National Health Promotion and Disease Prevention Objectives. Washington, D.C., Department of Human Health and Services, 1990, pp 571-578

17. Waller PF, Stewart JR, Hansen AR, Stutts JC, Popkin CL, Rodgman EA: The potentiating effects of alcohol on driver injury. JAMA 256:1461-1466, 1986

18. Waller JA: Methodological issues in hospital-based injury research. J Trauma 28:1632-1636, 1988

19. Chang G, Astrachan BM: The Emergency Department surveillance of alcohol intoxication after motor vehicle accidents. JAMA 260:2533-2536, 1988

20. Cherpitel CJS: Alcohol consumption and casualties: A comparison of two emergency room populations. Br $J$ Addict 83:1299-1307, 1992

21. Soderstrom CA, Dischinger PC, Smith GS, McDuff DR, Hebel RJ, Gorelick DA: Psychoactive substance dependence among trauma center patients. JAMA 267:2756-2759, 1992

22. Yates DW, Hadfield JM, Peters K: The detection of problem drinkers in the accident and emergency department. Br J Addict 82:163167,1987

23. Mason MF, Dubowschik M: Alcohol, traffic, and chemical testing in the United States: A resume and some remaining problems. Clin Chem 20:126-140, 1974

24. Coombs RH, Fawzy FI, Gerber BE: Patterns of cigarette, alcohol and other drug use among children and adolescents: A longitudinal study. Int J Addict 21:897-913, 1986 